Problem: Hat die Fourier-Algebra der freien Gruppe mit zwei Erzeugenden approximierende Einheiten? Oder allgemeiner: Gibt es lokal kompakte Gruppen, deren Fourier-Algebra keine approximierenden Einheiten besitzt (auch keine unbeschränkten)?

Für einige Verbesserungen und Hinweise bin ich P. Eymard sehr zu Dank verpflichtet.

\title{
Literatur
}

[1] P. Eymard, L'algèbre de Fourier d'un groupe tocalement oompaot, Bull. Soo. Math. France 92 (1964), S. 181-236.

[2] - Algèbres $A_{p}$ et convohutenrs de $L^{p}$, Séminaire Bourbaki, 220 annóe, 1969/70, $\mathrm{n}^{0} 367$.

[3] A. Figà-Talamanca et M. Picardello, Multiplicateurs de $A(G)$ qui ne sont pas dans $B(G)$, Comptes Rendus Acad. Sc. Paris, 9 juillot 1.973

[4] M. Lefranc, Comptes Rendus Ácad. Sc. Paris, 26 juin 1972.

[5] M. Leinert, Oonvoluteurs de groupes discrets, Comptes Rendus Acad. So Paris 271 (1970), S. $630-631$.

\section{Sums of independent Banach space valued random variables}

by

\section{JØRGEN IIOFFMANN JØRGENSEN (Åhus)}

Alsatract. Various generalizations of elassical thoorems about sums of independent real random variables to Banach space valued random variables are given: Let $\left(X_{n}\right)$ bo independent random variables with values in a Banach space, and let $\left(S_{n}\right)$ be their partial sums. Then necessary and sufficient conditions for $\left(S_{n}\right)$ to converge in $T^{p}$ is given $(0<p \leqslant \infty)$. For $p=\infty$ this gives a new characterization of those Banach spaces which do not contain $c_{0}$. Later we characterize the class of Banach spaces for which a.s. boundednoss of $\left(S_{n}\right)$ implies a.s. convergence of $\left(S_{n}\right)$. Finally we prove that convergence in distribution of $\left(S_{n}\right)$ in a weak topology of our Banach space implies a.s. norm-convergenco.

1. Introduction. We shall in this paper study the properties of series of independent random variables with values in a Banach space, that is, series of the form

$$
\sum_{n=1}^{\infty} X_{n}
$$

where $X_{1}, X_{2}, \ldots$ are independent random variables with values in a Banach space $E$.

Such series have been studied by Nordlander [13], Kahane [9], and Ito and Nisio [8].

Section 2 contains the basic definitions and notation, and we list some known lemmas and theorems for reference in later sections.

In Section 3 we study boundedness and convergence of (1.1) in $t^{p}(0 \leqslant p \leqslant \infty)$. Theorem 3.1 is a generalization of Theorem 4 , p. 17 in [9] and of Theorem 18.1.A, p. 254 in [12]. In [9] Kahane only considers the case $X_{n}=\varepsilon_{n} x_{n}$ where $\left(\varepsilon_{n}\right)$ is a Bernoulli sequence and $\left(x_{n}\right)$ is a non-random sequence of vectors. In [12] thotve only considers the case where $X_{n}$ is real and the $X_{n}$ 's are uniformly bounded. Theorem 3.1 gives new results even for real valued random variables. Theorem 3.5 and Corollary 3.7 give a new oharacterization, of those Banach spaces that do not contain $o_{0}$.

In Section 4: we show that eonvergence or boundedness of (1.1) may imply convergence or boundedness of

$$
\sum_{1}^{\infty} Y_{n}
$$


where $\left(Y_{n}\right)$ are independent Banach space valued random variables, such that $Y_{n}$ is a random multiple of $X_{n}$, with the multipliers satisfying certain conditions. In Theorem 4.3 we use an idea of $B$. Maurey (oral communication) to strengthen our original version considerably.

It is well known that if $X_{n}$ is symmetric and real for all $n \geqslant 1$, then boundedness of the partial sums of (1.1) implies convergence of (1.1). In Section 5 we show that the class of Banach spaces for which this holds is the class of Banach spaces for which $c_{0}$ is not contained in $L^{p}\left(I, \lambda, D^{\prime}\right)$ for some (or all) $1 \leqslant p<\infty$, where $I$ is the unit interval and $\lambda$ is the Lebesgue measure. I conjecture that if $c_{0} \subseteq L^{p}(I, \lambda, E)$ for some $1 \leqslant p<\infty$. then $E$ contains $c_{0}$. In Section 5 we show that relatively compactness of the partial sums of (1.1) in suitable topology $\tau$ on $D$ implies convergence of (1.1).

In Section 6 we prove that weak convergence in a weak topology of the partial sums of (1.1) implies a.s. norm-convergence of (1.1), if $\not X$ is symmetric. This result is closely related to that of Ito and Nisio [8]

2. Definitions and preliminary results. Let $E$ denote a Banach space with norm $\|\cdot\|$ and $(\Omega, \mathscr{F}, P)$ a probability space in all of this paper.

An $E$-valued random variable $X: \Omega \cap D$ is a $P$-measurable function in the sense of Definition III. 2,10 in [4]. That is,

$$
X^{-1}(A)=\{X \in A\} \in \mathscr{F} \quad \forall A \in \mathscr{B}(E),
$$

(2.2) $\exists E_{0}$ separable closed subspace of $D$ such that $P\left(X \in D_{0}\right)=1$

where $\mathscr{B}(E)$ is the Borel $\sigma$-algebra on $D$.

We shall for short denote $L^{p}(\Omega, \mathscr{F}, P, E)$ by $I^{p}(E)$ for $0 \leqslant p \leqslant \infty$. That is, if $0<p<\infty$, then $L^{p}(E)$ is the set of $E$-valued random variables, $X$, with

$$
\mathbf{E}\left(\|X\|^{p}\right)=\int_{\Omega}\|X(\omega)\|^{p} P(d \omega)<\infty
$$

where $\mathbf{E}$ denotes the expectation (= the integral with respect to $P$ ). $L^{0}(E)$ is the set of all $E$-valued random variables, and $L^{\infty}(E)$ is the set of $E$-valued random variables with ess sup $\|X(\omega)\|<\infty$. We define the metric $\|\cdot\|_{0}$ (norm if $1 \leqslant p \leqslant \infty$ ) by

$$
\begin{aligned}
& \|X\|_{0}=\mathbf{E}\left\{\frac{\|X\|}{1+\|X\|}\right\}, \quad \forall X \in L^{0}(E), \\
& \|X\|_{p}=\mathbf{E}\left(\|X\|^{p}\right), \\
& \|X\|_{p}=\left\{\mathbf{E}\|X\|^{p}\right\}^{1 / p} \\
& \forall \dot{X} \in L^{p}(E), \forall 0<p \leqslant 1, \\
& \forall X \in L^{p}(\mathbb{E}), \forall 1 \leqslant p<\infty \text {, } \\
& \|X\|_{\infty}=\operatorname{ess} \sup \|X(\omega)\|, \quad \forall X \in L^{\infty}(E) .
\end{aligned}
$$

Then $L^{p}(E)$ is a Fréchet space for all $0 \leqslant p \leqslant \infty$, and if $1 \leqslant p \leqslant \infty$ then
It is well known that we have:

PropostTton 2.1. If $K \subseteq L^{p}(E), 0<p \leqslant \infty$, then $K$ is a bounded subset of $L^{p}(\mathbb{E})$ if and only if

$$
\sup \left\{\|X\|_{p}: X \in K\right\}<\infty .
$$

If $K \subseteq L^{0}(E)$, then the following 3 statements are equivalent:

$K$ is a bounded subset of $L^{0}(E)$,

(2.6) $\forall \varepsilon>0, \exists K<\infty$, such that $P(\|X\| \geqslant K) \leqslant \varepsilon \forall X \in K$.

If $\left(X_{n}\right)$ is a sequence in $L^{0}(D)$, then $\left(X_{n}\right)$ converges in $L^{0}(E)$ to $X$ if and only if

$$
P\left(\left\|X_{n}-X\right\| \geqslant \varepsilon\right) \underset{n \rightarrow \infty}{\longrightarrow} 0 \forall \varepsilon>0 .
$$

If $X_{n} \underset{n \rightarrow \infty}{\longrightarrow} X$ in $L^{0}(E)$ wo shall say that $\left(X_{n}\right)$ converges in probability to $X$. If $K$ is a bounded subset of $L^{0}(E)$ we shall say that $K$ is stochastically bounded.

Let $(H, \mathscr{H})$ be a measurable space, that is, a set $H$ and a $\sigma$-algebra $\mathscr{H}$. If $X: S \frown H$ is a measurable map, then the image measure of $P$ under $X$ is denoted by $P_{X^{x}}$ and oalled the distribution law of $X$. That is,

$$
P_{X}(A)=P(X \in A) \quad \forall A \in \mathscr{H} \text {. }
$$

An $I$-valued random variable $X$ is called symmetric if $P_{X}=P_{-X}$, and a sequence $\left(X_{n}\right)$ is called a symmetric sequence if $P_{\boldsymbol{X}}=P_{\boldsymbol{Y}}$ for all $\boldsymbol{Y}=\left( \pm X_{n}\right)$ with an arbitrary choice of $上$, where $\boldsymbol{X}=\left(X_{n}\right)$; (here $\boldsymbol{X}$ and $\boldsymbol{Y}$ are considered measurable maps from $\Omega$ into $E^{\infty}$, with its product $\sigma$-algebra: $\mathscr{B}(E) \otimes \mathscr{B}(H) \otimes \ldots)$.

It is alear that if $X_{1}, X_{2}, \ldots$ are symmetric independent $E$-valued random variables, then $\left(X_{n}\right)$ is a symmetric sequence.

If $X$ is an $E$-valued random variable, then $X^{*}$ is called a symmetrization of $X$, is $X^{*}=X-X^{\prime}$, where $P_{X}=P_{X^{\prime}}$ and $X$ and $X^{\prime}$ are independent. Tt $\left(X_{n}\right)$ is a sequence of $H$-valued random. variables then $\left(X_{n}^{*}\right)$ is called a symmetrization of $\left(X_{n}\right)$ if $X_{n}^{*}=X_{n}-X_{\psi_{0}}^{\prime}$ where $\left(X_{n}\right)$ and $\left(X_{n}^{\prime}\right)$ are independent and equidistributed. A symmetrization of $\left(X_{n}\right)$ will always exist wi least on the product probability space $(\Omega \times \Omega, \mathscr{F} \otimes \mathscr{F}$, $P \times P)$. If $\left(X_{n}\right)$ is a sequonce of independent random variables, and $\left(X_{n}^{*}\right)$ is a symmetrization of $\left(X_{n}\right)$, then it is easily seen that $\left(X_{n}^{*}\right)$ is a symmetric sequence.

If $\left(\varepsilon_{j}\right)$ are independent random variables, so that $p\left(\varepsilon_{j}=1\right)=p\left(\varepsilon_{j}\right.$ $=-1)=\frac{1}{2}$ for all $j \geqslant 1$, then $\left(\varepsilon_{j}\right)$ is called a Bernoulli sequence, and we 
define

$$
\begin{aligned}
& C(E)=\left\{\left(x_{j}\right) \in E^{\infty} \mid \sum_{1}^{\infty} \varepsilon_{j} x_{j} \text { converges in } L^{0}(E)\right\}, \\
& B(E)=\left\{\left(x_{j}\right) \in E^{\infty} \mid\left(\sum_{1}^{\infty} \varepsilon_{j} x_{j}\right)_{n=1}^{\infty} \text { is bounded in } L^{0}(\mathbb{E})\right\} .
\end{aligned}
$$

It follows from Theorem 3.1 that we may substitute $L^{0}(E)$ with $L^{p}(E)$ for all $0 \leqslant p<\infty$ in the definition of $O(E)$ and $B(E)$.

The following two propositions are well. known (see, for example, Lemma 1, p. 12 in [9] and 17.1.A, p. 245 in [12]).

THEOREM 2.2. If $X^{*}$ is a symmetrization of $X$ and $a \in \mathbb{E}$, then we have

$$
P\left(\left\|X^{*}\right\| \geqslant t\right) \leqslant 2 P\left(\|X-a\| \geqslant \frac{1}{2} t\right), \quad \forall t \geqslant 0 .
$$

THEOREM 2.3. Let $\left(X_{j}\right)$ be a symmetric sequence and put $S_{n}=X_{1}+$ $+\ldots+X_{n}$. If $M$ is an infinite subset of $\{1,2, \ldots\}$, then

$$
\begin{array}{cc}
P\left(\max _{1 \leqslant j \leqslant n}\left\|S_{j}\right\| \geqslant t\right) \leqslant 2 P\left(\left\|S_{n}\right\| \geqslant t\right) & \forall t \geqslant 0, \\
P\left(\sup _{j}\left\|S_{j}\right\| \geqslant t\right) \leqslant 2 \sup _{n \in M} P\left(\left\|S_{n}\right\| \geqslant t\right) & \forall t \geqslant 0 .
\end{array}
$$

If $S_{n}$ converges in probability to $S$ then

$$
P\left(\sup _{j}\left\|S_{j}\right\| \geqslant t\right) \leqslant 2 P(\|S\| \geqslant t) \quad \forall t \geqslant 0 .
$$

A slight modification of the proofs of Theorem 1 and Theorem 2, p. 11 in [9] gives us:

THEOREM 2.4. Let $\left(X_{n}\right)$ be a symmteric sequenee and $S_{n}=X_{1}+\ldots+X_{n}$, then the following 3 conditions are equivalent:

(2.11) $\left(S_{n}\right)$ has a subsequence which is convergent in probability (stochastically bounded),

(2.12) $\left(S_{n}\right)$ is convergent in probability (stochastically bounded),

(2.13) $\left(S_{n}\right)$ is convergent a.s. (bounded a.s.).

THEOREM 2.5. Let $\left(X_{j}\right)$ be independent E-valued random variables and put $S_{n}=X_{1}+\ldots+X_{n}$. Then $\left(S_{n}\right)$ is convergent in probability (stoohas tically bounded) if and only if $\left(S_{n}\right)$ is convergent a.s. (bounded a.s.).

Let us conclude this section with the following simple but useful propositions:

THorem 2.6. Let $X$ and $Y$ be two independent random variables both belonging to $L^{p}(E)$ for some $0 \leqslant p \leqslant \infty$.

If either. $X$ has mean 0 and $p \geqslant 1$, or $X$ is symmetric, then we have

$$
\frac{1}{2}\|2 Y\|_{p} \leqslant\|X+Y\|_{p} \text {. }
$$

In partioular,

$$
\begin{gathered}
2^{p-1}\|Y\|_{p} \leqslant\|X+Y\|_{p} \quad \text { for } \quad 0<p \leqslant 1, \\
\|Y\|_{p} \leqslant\|X+Y\|_{p} \text { for } \quad 1 \leqslant p \leqslant \infty .
\end{gathered}
$$

The proof is easy and we leave the verification to the reader.

Puoposition 2.7. Let $X$ be an E-valued random variable and $\varphi$ an increasing continuous function on $[0, \infty)$. Let $R(t)$ be the tail probability function:

$$
R(t)=P(\|X\| \geqslant t), \quad t \geqslant 0 .
$$

Then $\varphi(\|X\|)$ is integrable if and only if $R(t)$ is Lebesgue-Stieltjes integrable with respect to $\phi$, and then voe have

$$
\mathbf{E}(\varphi(\|X\|))=\varphi(0)+\int_{0}^{\infty} R(t) d \varphi(t) .
$$

This is simply integration by parts!

Proposturon 2.8. Let $\left(X_{j}\right)$ be a symmetric sequence; then we have

$$
\begin{gathered}
P\left(\sum_{1}^{\infty} X_{j} \text { converges }\right)=P\left(\left(X_{j}\right) \in O(E)\right), \\
P\left(\left(\sum_{i}^{n} X_{j}\right)_{n=1}^{\infty} \text { is bounded }\right)=P\left(\left(X_{j}\right) \in B(E)\right) .
\end{gathered}
$$

This follows easily from Fribini's theorem and the fact that $\left(X_{j}\right)$ and $\left(\varepsilon_{j} X_{j}\right)$ are equidistributed if $\left(\varepsilon_{j}\right)$ is a Bernoulli sequence, which is independent of $\left(X_{j}\right)$.

3. Convergence and boundedness of $\left(S_{n}\right)$ in $L^{P}(E)$. Let $\left(X_{n}\right)$ be a sequence of independent to-valued random variables, and put

$$
S_{n}=\sum_{j=1}^{n} X_{j} ; \quad N=\sup _{n}\left\|X_{n}\right\| ; \quad M T=\sup _{n}\left\|S_{n}\right\| .
$$

We shall in this section study convergence and boundedness of $\left(S_{n}\right)$ in $L^{p}(E)(0 \leqslant p \leqslant \infty)$. Wo know wlready from Theorem 2.5 that convergence or boundedness of $\left(S_{n}^{\prime}\right)$ in $L^{p}(H)$ implies a.s. convergence respectively a.s. boundedness of $\left(S_{n}\right)$. Our tirst theorem tells us when the converse holds.

Theorism 3.1. Let $\left(X_{j}\right)$ be independent tevalued random variables such that
$\left(S_{n}\right)$ is stochastioally bounded,

$$
N \in L^{p}(\boldsymbol{R}),
$$

for some $0<p<\infty$. Then $M \in L^{p}(\boldsymbol{R})$. 
Proof. Notice first that $M<\infty$ a.s. by Theorem 2.5 , and $N \leqslant 2 M$. $<\infty$. Let us first assume that $X_{j}$ is symmetric for $j \geqslant 1$.

Let $R_{k}(t)=P\left(\left\|S_{k}\right\| \geqslant t\right), R(t)=P(M \geqslant t)$ and $Q(t)=P(N \geqslant t)$. We shall then prove that

$$
R_{k_{0}}(2 t+s) \leqslant Q(s)+4 R_{k_{0}}(t)^{2} \quad \forall t, s \geqslant 0 \quad \forall k_{i} \geqslant 1 .
$$

Let $T$ be the stopping time defined by

$$
T=\inf \left\{n \geqslant 1 \mid\left\|S_{n}\right\| \geqslant t\right\}
$$

where $\inf (\varnothing)=\infty$. Then $\left\|S_{t}\right\| \geqslant 2 t+s$ implies that $T \leqslant 7$, and so we have

$$
R_{k}(2 t+s)=\sum_{j=1}^{h} P\left(\left\|S_{k}\right\| \geqslant 2 t+8, T=j\right) .
$$

If $T=j$ and $\left\|S_{k}\right\| \geqslant 2 t+s$ then $\left\|S_{j-1}\right\|<t$, and so

$$
\left\|S_{k}-S_{j}\right\| \geqslant\left\|S_{k}\right\|-\left\|S_{j-1}\right\|-\left\|X_{j}\right\| \geqslant t+s-N \text {. }
$$

Hence we have

$$
\begin{aligned}
P\left(T=j,\left\|S_{k}\right\| \geqslant 2 t+s\right) & \leqslant P\left(T=j,\left\|S_{k}-S_{j}\right\| \geqslant t+s-N\right) \\
& \leqslant P(T=j, N \geqslant s)+P\left(T=j,\left\|S_{k}-S_{j}\right\| \geqslant t\right) \\
& =P(T=j, N \geqslant s)+P(T=j) P\left(\left\|S_{l_{c}}-S_{j}\right\| \geqslant t\right)
\end{aligned}
$$

since $\{T=j\}$ and $\left\{\left\|S_{k}-S_{j}\right\| \geqslant t\right\}$ are independent events. Hence we have that

$$
R_{k}(2 t+s) \leqslant Q(s)+\sum_{j=1}^{k} P(T=j) P\left(\left\|S_{k}-S_{j}\right\| \geqslant t\right) .
$$

Now let $Y_{1}=S_{k}-S_{j}$ and $Y_{2}=S_{j}$; then $Y_{1}$ and $Y_{2}$ are symmetric independent random variables and $Y_{1}+Y_{2}=S_{l 6}$, so by Theorem 2.3 we have that

$$
P\left(\left\|S_{k}-S_{j}\right\| \geqslant t\right) \leqslant 2 P\left(\left\|S_{l}\right\| \geqslant t\right),
$$

and since $\{T \leqslant k\}=\left\{\underset{1 \leqslant j \leqslant k}{\max }\left\|S_{j}\right\| \geqslant t\right\}$, we have that

$$
\begin{aligned}
R_{k}(2 t+s) & \leqslant Q(s)+2 R_{k}(t) \sum_{j=1}^{l} P(T=j) \\
& =Q(s)+2 R_{k}(t) P\left(\max _{1=j \leqslant k}\left\|S_{j}\right\| \geqslant t\right) \\
& \leqslant Q(s)+4 \cdot R_{k}(t)^{2},
\end{aligned}
$$

where we have used Theorem 2.3. Hence (3.3) is proved. From (3.1) and Theorem 2.3 we find that

since $R_{k}(t) \leqslant \dot{R(t)} \leqslant 2 \sup _{k} R_{\gamma_{k}}(t)$.
Now let $t_{0}>0$ be chosen so that $R\left(t_{0}\right)<\frac{1}{16 \cdot 3^{p}}$. If $A>3 t_{0}$ we then

$$
\begin{aligned}
\int_{0}^{A} p x^{p-1} R(x) d x & =3^{p} \cdot p \int_{0}^{A / 3} x^{p-1} R(3 x) d x \\
& \leqslant 3^{p} \cdot p \cdot 2 \int_{0}^{A / 3} x^{p-1} Q(x) d x+8 p 3^{p} \int_{0}^{A / 3} x^{p-1} R(x)^{2} d x \\
& \leqslant 2 \cdot 3^{p} \mathbf{E}\left(N^{p}\right)+8 \cdot 3^{p} \cdot t_{0}^{p} \cdot p+8 p 3^{p} \int_{0}^{A / 3} x^{y-1} R\left(t_{0}\right) R(x) d x \\
& \leqslant O+\frac{1}{2} \int_{0}^{A} p x^{p-1} R(x) d x
\end{aligned}
$$

where $C=2 \cdot 3^{p} \mathbf{E}\left(N^{p}\right)+8 \cdot 3^{p} \cdot t_{0}^{p}$, where we have used Lemma 2.7. Hence

$$
\int_{0}^{A} p x^{p-1} R(x) d x \leqslant 2 O \quad \forall A>3 t_{0}
$$

and so $M \in L^{p}(E)$ by Lemma 2.7 .

If $\left(X_{j}\right)$ are not symmetric, then we consider a symmetrization $X_{j}^{*}$ $=\left(X_{j}-X_{j}^{\prime}\right)$ of $\left(X_{j}\right)$. Let us define

$$
\begin{array}{ll}
S_{n}^{\prime}=\sum_{j=1}^{n} X_{j}^{\prime} ; & S_{n}^{*}=\sum_{j=1}^{n} X_{j}^{*}=S_{n}-S_{n}^{\prime} ; \\
M^{\prime}=\sup _{n}\left\|S_{n}^{\prime}\right\| ; & M^{*}=\sup _{n}\left\|S_{n}^{*}\right\| \leqslant M+M^{\prime} ; \\
N^{\prime}=\sup _{n}\left\|X_{n}^{\prime}\right\| ; & N^{*}=\sup _{n}\left\|X_{n}^{*}\right\| \leqslant N+N^{\prime} .
\end{array}
$$

Since $M$ and $M^{\prime}$ are equidistributed and $N$ and $N^{\prime}$ are equidistributed, we find that $M^{*}<\infty$ a.s., and $N^{*} \in L^{p}(Z)$. Thus from the argument above we have that $M^{*} \in L^{p}(E)$, and so

$$
\infty>\mathbf{E}\left\{\left(M^{*}\right)^{p}\right\}=\int_{k^{\prime \infty}} \mathbf{E}\left\{\sup _{n}\left\|S_{n}-\sum_{j=1}^{n} x_{j}\right\|^{p}\right\} \mu(d x),
$$

where $\mu$ is the distribution law of $\left(X_{n}^{\prime}\right)$. Now since $\left(S_{n}^{\prime}\right)$ is bounded a.s., we can find $\left(x_{j}\right) \in D^{\infty}$ so that

$$
\begin{gathered}
o=\sup _{n}\left\|\sum_{j=1}^{n} x_{j}\right\|<\infty, \\
\mathbf{E}\left\{\left(\sup _{n}\left\|S_{n}-s_{n}\right\|\right)^{p}\right\}<\infty,
\end{gathered}
$$


where $s_{n}=x_{1}+\ldots+x_{n}$. Now

$$
M \leqslant c+\sup _{n}\left\|S_{n}-s_{n}\right\|,
$$

and so $M \epsilon L^{p p}(E)$, which proves the theorem.

CoRollary 3.2. Let $\left(X_{j}\right)$ be independent $t$-valued random variables, so that $\left(S_{n}\right)$ is stochastically bounded, and let $0<p<\infty$. Then the following 3 statements are equivalent:

$$
\begin{array}{cc}
(3.4) & \left(S_{n}\right) \text { is bounded in } L^{p}(\mathbb{H}), \\
(3.5) & M=\sup _{n}\left\|S_{n}\right\| \in L^{p}(\boldsymbol{R}) \\
(3.6) & N=\sup _{n}\left\|X_{n}\right\| \in L^{p}(\boldsymbol{R}) .
\end{array}
$$

Furthermore $\left(S_{n}\right)$ is bounded in $L^{\infty}(E)$ if and only if $M \in L^{\infty}(\boldsymbol{R})$.

Proof. The last statement is obvious.

We know from Theorem 3.1 that (3.6) implies (3.5), and (3.5) obviously implies (3.4) and (3.6). Hence it suffices to prove that (3.4) implies (3.5). To do this we shall first assume that $X_{j}$ is symmetric for all $j \geq 1$.

Let $R(t)=P(M \geqslant t), R_{k}(t)=P\left(\left\|S_{k}\right\| \geqslant t\right)$; then by Theorem 2.3 we have

$$
R(t) \leqslant 2 \liminf _{k \rightarrow \infty} R_{k}(t) \quad \forall t \geqslant 0 .
$$

Now let $A>0$; then by Fatou's lemma we have

$$
\begin{aligned}
\int_{0}^{A} p x^{p-1} R(x) d x & \leqslant 2 \liminf _{k \rightarrow \infty} \int_{0}^{A} p x^{p-1} R_{k}(x) d x \\
& \leqslant 2 \liminf _{k \rightarrow \infty}\left\|S_{k}\right\|^{p} \\
& <\infty .
\end{aligned}
$$

Thus, by Lemma 2.7, we have that $\mathbf{E}\left(M^{p}\right)<\infty$. The general case follows by a symmetrization procedure similar to the one in the last part of Theorem 3.1.

CoRoLLaRr 3.3. Let $\left(X_{j}\right)$ be independent E-valued random variables, so that $\left(S_{n}\right)$ converges a.s. to $S$, and let $0<p<\infty$. Then the following 5 statements are equivalent:

$$
\begin{aligned}
& S \in L^{p}(I), \\
& M \in L^{p}(\boldsymbol{R}), \\
& N \in L^{p}(\boldsymbol{R}),
\end{aligned}
$$$$
S_{n} \rightarrow S \text { in } L^{p}(\mathscr{D})
$$

Furthermore $S \in L^{\infty}(E)$ if and only if $M \in L^{\infty}(E)$.
Remark. It is easy to construct an example where $S$ belongs to $L^{\infty}\left(c_{0}\right)$, but $S_{n} \rightarrow S$ in $L^{\infty}\left(c_{0}\right)$. However, in Theorem 3.5 we shall prove that if $S \in L^{\infty}(E)$ and $E$ does not contain $c_{0}$, then $S_{n} \rightarrow S$ in $L^{\infty}(E)$.

Proof. (3.9), (3.1.0) and (3.1.1) are equivalent by Corollary 3.2, (3.7) obviously implies (3.8), and (3.9) implies (3.7) (use Lebesgue's theorem on dominated convergence). Hence it suffices to prove that (3.8) implies (3.9). To do this we shall assume that $X_{j}$ is symmetric for all $j \geqslant 1$. In this case we have by Theorem 2.3 that

$$
P(M \geqslant t) \leqslant 2 P(\|S\| \geqslant t),
$$

and so il $\& \in L^{p}(E)$ then by Lemma 2.7 we have that $M \in L^{p}(\boldsymbol{R})$ (note that this result also holds for $p=\infty)$.

Hence (3.8) implies (3.9), and we have also proved the last part of Corollary 3.3 under the assumption of symmetry of $X_{j}$. The general case is proved by a standard symmetrization procedure.

Coxoriraxx 3.4. Let $\left(X_{j}\right)$ be independent $E$-valued random variables and $\left(a_{j}\right)$ a decreasing sequence of non-negative real numbers. Let

$$
U_{n}=a_{n} \sum_{j=1}^{n} X_{j} ; \quad V=\sup _{n}\left\|U_{n}\right\| ; \quad W=\sup _{n}\left\|a_{n} X_{n}\right\| .
$$

Suppose that $V<\infty$ a.s. Then $W<\infty$ a.s., and if $W \in L^{p}(\boldsymbol{R})$ for some $0<p<\infty$, then $V \in L^{p}(\boldsymbol{R})$.

Proof. Let us define

$$
\tilde{X}_{j}=\left(0, \ldots, 0, a_{j} X_{j}, a_{j+1} X_{j}, \ldots, a_{n} X_{j}, \ldots\right) .
$$

Then $\tilde{X}_{j}$ is a $l^{\infty}(E)$-valued random variable, such that $\tilde{X}_{1}, \tilde{X}_{2}, \ldots$ are independent, and.

$$
\left\|\tilde{X}_{j}(\omega)\right\|_{\infty}=a_{j}\left\|X_{j}(\omega)\right\| \quad \forall j \geqslant 1, \forall \omega \in \Omega .
$$

If $\tilde{S}_{j}=\tilde{X}_{1}+\ldots+\tilde{X}_{j}$ and $s_{j l}$ is the $k$ th coordinate of $\tilde{S}_{j}$, then

$$
S_{j / k}= \begin{cases}a_{k} \sum_{v=1}^{j} X_{v} & \text { if } j<k, \\ U_{k} & \text { if } j \geqslant k .\end{cases}
$$

Now since $a_{k}=a_{j}$ tor $j<k$, we find that

$$
\left\|\tilde{S}_{j}(\omega)\right\|_{\infty}=\sup _{l c}\left\|S_{j k}(\omega)\right\|=\max _{1 \leqslant k \leqslant j}\left\|U_{k_{k}}\right\| \leqslant V(\omega) .
$$

Hence $\left(\tilde{S}_{j}(\omega)\right)$ is a.k. bounded and Corollary 3.4 is an immediate consequence of: Theorem 3.1. 
THeonem 3.5. Let $\left(X_{j}\right)$ be independent E-valued random variables such that the series

$$
S=\sum_{j=1}^{\infty} X_{j}
$$

converges a.s. and $S \in L^{\infty}(E)$. If $E$ does not contain a subspace isomorphio to $c_{0}$, then the series (3.12) converges in $L^{\infty}(E)$.

Proof. Suppose that $S \in L^{\infty}(E)$. From Corollary 3.3 it follows that

$$
s_{n}=\sum_{j=1}^{n} \mathbf{E} X_{j}
$$

converges to ES. Let $X_{j}^{\prime}=X_{j}-\mathbf{E} X_{j}$ and put $S_{n}^{\prime}=X_{1}^{\prime}+\ldots+X_{n}^{\prime}$. Then $S_{n}^{\prime} \underset{n \rightarrow \infty}{\longrightarrow} S^{\prime}=S-\mathbf{E} S$ a.s., $S^{\prime} \in L^{\infty}(E)$, and $S_{n \rightarrow S^{\prime}}^{\prime}$ in $L^{\infty}(E)$ if and only if $S_{n} \rightarrow S$ in $L^{\infty}(E)$.

This shows that it is no loss of generality assuming that $\mathbf{E} X_{j}=0$ for all $j \geqslant 1$, which we shall assume for the rest of the proof.

Now suppose that $S_{n} \rightarrow S$ in $L^{\infty}(E)$; we shall then show that $E$ contains a closed subspace isomorphic to $c_{0}$. Since $S_{n} \rightarrow S$ a.s., we see that $\left(S_{n}\right)$ is not a Cauchy sequence in $L^{\infty}(E)$. Hence we can find $a>0$ and a sequence of integers: $0=n_{0}<n_{1}<\ldots$ such that (put $S_{0}=0$ )

$$
\left\|S_{n_{j+1}}-S_{n_{j}}\right\|_{\infty}>a \quad \forall j=0,1, \ldots
$$

Let $Y_{j}=S_{n_{j+1}}-S_{n_{j}}$ for $j=0,1, \ldots$ Then

$$
\sum_{j=0}^{k} Y_{j}=S_{n_{k}}
$$

and so $\sum_{0}^{\infty} Y_{j}=S$ a.s. Now $Y_{j}$ takes values in a bounded separable subset of $E$ with probability 1 . Hence we can find random variables $Z_{j}$ such that $\mathbf{E} Z_{j}=\mathbf{E} \bar{Y}_{j}=0$ and

$$
\begin{gathered}
Z_{j}=\sum_{v=1}^{\infty} x_{j v} 1_{A_{j v}} \quad \forall j \geqslant 0, \\
\left\|Z_{j}-Y_{j}\right\|_{\infty} \leqslant a 2^{-j-1} \quad \forall j \geqslant 0,
\end{gathered}
$$

where $\left\{A_{j v} \mid v=1,2, \ldots\right\}$ are disjoint sets in the $\sigma$-algebra $X_{j}^{-1}(\mathscr{B}(\mathbb{X}))$ and $x_{j v} \in \mathbb{E}$. Since

$$
\sum_{j=1}^{\infty}\left\|Z_{j}-Y_{j}\right\|_{\infty} \leqslant a<\infty,
$$

we find that $\sum_{0}^{\infty} Z_{j}=T$ exist a.s. and $T \epsilon L^{\infty}(E)$. Also we have that

$$
\left\|Z_{j}\right\|_{\infty} \geqslant\left\|Y_{j}\right\|_{\infty}-a 2^{-j-1}>\frac{1}{2} a \quad \forall j \geqslant 0 .
$$

Hence we can find $v(j) \geqslant 1$ such that $\left\|x_{j v(j)}\right\| \geqslant \frac{1}{2} a$ and $P\left(A_{j v(j)}\right)>0$. Let us put $y_{j}=x_{j v(j)}$ and $B_{j}=A_{j v(j)}$ for $j \geqslant 0$.

Since $Y_{0}, Y_{1}, \ldots$ are independent random variables, we have that $Z_{0}, Z_{1}, \ldots$ are independent and so, by Theorem 3.5 , we can find a constant $K>0$ such that

$$
\left\|\sum_{j=1}^{n} Z_{j}\right\|_{\infty} \leqslant K \quad \forall n \geqslant 1 .
$$

Since $\mathbf{E} Z_{j}=0$, it follows from Theorem 2.6 that whenever $\sigma \subseteq\{0, \ldots, n\}$, then we have

$$
\left\|\sum_{y \in \sigma} Z_{y}\right\|_{\infty} \leqslant\left\|\sum_{j=1}^{n} Z_{y}\right\|_{\infty} \leqslant K .
$$

That is, there exists an event $\Omega_{0} \subseteq \Omega$ with $P\left(\Omega_{0}\right)=1$ and

$$
\left\|\sum_{j \in \sigma} Z_{j}(\omega)\right\| \leqslant K \quad \forall \omega \in \Omega_{0}
$$

and for all finite subsets $\sigma$ of $\{0,1, \ldots\}$ (there is at most countably many finite subsets of $\{0,1, \ldots\})$.

We know that $P\left(B_{j}\right)>0$ and that $B_{0}, \ldots, B_{n}, \ldots$ are independent. Hence we have that $P\left(\bigcap_{0}^{n} B_{j}\right)>0$ and so we can find an $\omega_{n} \in \Omega_{0} \cap B_{0} \cap \ldots \cap B_{n}$. Since $Z_{j}\left(\omega_{n}\right)=y_{j}$ for $0 \leqslant j \leqslant n$, we find that

$$
\left\|\sum_{j \in \sigma} y_{1}\right\| \leqslant K, \quad \sigma \subseteq\{1, \ldots, n\} \quad \forall n \geqslant 1
$$

But this implies that

$$
\sum_{j=1}^{\infty}\left|\left\langle x^{\prime}, y_{j}\right\rangle\right| \leqslant 2 K\left\|x^{\prime}\right\| \quad \forall x^{\prime} \in E^{\prime},
$$

and since $\left\|y_{j}\right\| \geqslant \frac{1}{2} a$, it follows from Theorem 5 in [1] that $\mathbb{E}$ contains a subspace isomorphic to $o_{0}$.

Troforwar 3.6. Let $\left(X_{j}\right)$ be a sequence of independent integrable $E$-valued random variables, with $\mathbf{E} X_{j}=0$ for all $j \geqslant 1$. Then the following 4 conditions are equivalent:

$$
\left(\sum_{i}^{n} X_{j}\right) \text { is bounded in } L^{\infty}(\mathbb{E})
$$

(3.14) $\exists K>0, \exists \Omega_{0} \in \mathscr{F}$, such that $P\left(\Omega_{0}\right)=1$ and

$$
\sum_{j=1}^{\infty}\left|\left\langle x^{\prime}, X_{j}(\omega)\right\rangle\right| \leqslant K\left\|x^{\prime}\right\| \quad \forall x^{\prime} \in \mathbb{E}^{\prime}, \forall \omega \in \Omega_{0},
$$


(3.15) $\forall x^{\prime} \in E^{\prime}, \exists K\left(x^{\prime}\right)>0$, such that $P\left(\omega\left|\sum_{j=1}^{\infty}\right|\left\langle x^{\prime}, X_{j}(\omega)\right\rangle \mid \leqslant K\left(x^{\prime}\right)\right)=1$,

(3.16) $\forall x^{\prime} \in E^{\prime}, \exists K\left(x^{\prime}\right)>0$, such that $P\left(\omega|| \sum_{j=1}^{n}\left\langle x^{\prime}, X_{j}(\omega)\right\rangle \mid \leqslant K\left(x^{\prime}\right)\right)$ $=1 \quad \forall n \geqslant 1$.

If integrability of $X_{j}$ and $\mathbf{E} X_{j}=0$ is not assumed then (3.14) and (3.15) are equivalent, (3.15) implies (3.16), and (3.16) is equivalent to (3.1.3).

Proof. Suppose that (3.13) holds and $\mathbf{E} X_{j}=0$. Let $K$ be a finite constant such that

$$
\left\|\sum_{j=1}^{n} X_{j}\right\|_{\infty} \leqslant K
$$

Then, by Theorem 2.6, we have that

$$
\left\|\sum_{j \in \sigma} X_{j}\right\|_{\infty} \leqslant K
$$

for every finite subset $\sigma$ of $\{1,2, \ldots\}$. Since there is only countably many finite subsets of $\{1,2, \ldots\}$, we can find $\Omega_{0} \in \mathscr{F}$ with $P\left(\Omega_{0}\right)=1$ and

$$
\left\|\sum_{j \in \sigma} X_{j}(\omega)\right\| \leqslant K \quad \forall \omega \in \Omega_{0} .
$$

Hence we have

$$
\sum_{j=1}^{\infty}\left|\left\langle x^{\prime}, X_{j}(\omega)\right\rangle\right| \leqslant 2 K\left\|x^{\prime}\right\| \quad \forall \omega \in \Omega_{0}, \forall x^{\prime} \in \mathbb{H}^{\prime} .
$$

Now let us drop the assumptions on integrability of $X_{j}$ and $\mathbf{E} X_{j}=0$ for the rest of this proof.

It is evident that (3.14) implies (3.15), (3.15) implies (3.16), and (3.13) implies (3.16).

Suppose that (3.16) holds. Since $X_{j}$ is essentially separably valued for all $j \geqslant 1$, it is no loss of generality to assume that $E$ is separable. Let $U^{\prime}$ be the unit ball in $E^{\prime}$ equipped with the $w^{*-t o p o l o g y . ~ T h e n ~} U^{\prime}$ is metriz-

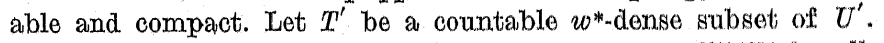

We know by assumption that $\left\langle x^{\prime}, S_{n}\right\rangle$ belongs to $T^{\infty}(\boldsymbol{R})$ for all $x^{\prime} \in \mathbb{T}^{\prime}$, hence we may define the linear map

$$
A_{n} x^{\prime}=\left\langle x^{\prime}, s_{n}\right\rangle=\sum_{j=1}^{n}\left\langle x^{\prime}, X_{\cdot j}\right\rangle
$$

from $E^{\prime}$ into $L^{\infty}(\boldsymbol{R})$. If $x_{k}^{\prime} \rightarrow x^{\prime}$ and $A_{n} x_{k}^{\prime} \rightarrow f$ in $L^{\infty}(\boldsymbol{R})$, then

$$
\left\langle x_{k}^{\prime}, S_{n}(\omega)\right\rangle \underset{k \rightarrow \infty}{\longrightarrow}\left\langle x^{\prime}, S_{n}(\omega)\right\rangle \quad \forall \omega \in \Omega
$$

and so $f=A_{n} x^{\prime}$. Hence $A_{n}$ is continuous by the closed graph theorem. By assumption. we know that $\sup \left\|A_{n} \alpha^{\prime}\right\|_{\infty}<\infty$ for all $x^{\prime} \in \mathbb{E}^{\prime}$, so by the principle of uniform boundedness we have that there exists a finite constant $K$ such that

$$
P\left(\left|\left\langle\infty^{\prime}, S_{n}\right\rangle\right| \leqslant K\right)=1 \quad \forall n \geqslant 1, \forall\left\|x^{\prime}\right\| \leqslant 1 .
$$

Since $T^{\prime}$ is countable, we can therefore find $\Omega_{0} \in \mathscr{F}$, with $P\left(\Omega_{0}\right)=1$ and

$$
\left|\left\langle x^{\prime}, \mathbb{S}_{n}(\omega)\right\rangle\right| \leqslant K \quad \forall x^{\prime} \in T^{\prime}, \forall \omega \in \Omega_{0} .
$$

It is well known that we can find compact subsets $K_{n m}$ of. $E$ such that

$$
P\left(S_{n} \in K_{n m}\right) \geqslant 1-2^{-n-m}
$$

because $D$ is a Polish space. Now let

$$
\Omega_{1}=\bigcup_{m=1}^{\infty} \bigcap_{n=1}^{\infty}\left\{S_{n} \in K_{n m}\right\} \cap \Omega_{0}
$$

it is then easily seen that $P\left(\Omega_{1}\right)=1$.

Let us take an $x^{\prime} \in U^{\prime}$ and an $\omega \in \Omega_{1}$. Then by Banach-Dieudonné's theorem (see, for example, [10], 21.10 (1), p. 272) there exists $y_{n m}^{\prime} \in T^{\prime}$ such that

$$
\left|\left\langle x^{\prime}-y_{n m}^{\prime}, \infty\right\rangle\right| \leqslant 1 \quad \forall x \in K_{n m}, \forall n, m .
$$

Since $\omega \in \Omega_{1}$, we can find an integer $m \geqslant 1$ with $\omega \in \Omega_{0} \cap\left\{S_{n} \in K_{n m}\right\}$ for all $n \geqslant 1$. Hence we have

$$
\left|\left\langle x^{\prime}, S_{n}(\omega)\right\rangle\right| \leqslant\left|\left\langle y_{n m}^{\prime}, S_{n}(\omega)\right\rangle\right|+\left|\left\langle x^{\prime}-y_{n m}^{\prime}, S_{n}(\omega)\right\rangle\right| \leqslant K+1
$$

since $\omega \in \Omega_{0}$ and $S_{n}(\omega) \in K_{n m}$. But this shows that $\left\|S_{n}(\omega)\right\| \leqslant K+1$ for $\omega \in \Omega_{1}$, and so (3.16) implies (3.13).

Let us then prove that (3.15) implies (3.14) (without the assumption that $\mathbf{E} X_{j}=0$ ). We have already proved that (3.15) implies (3.13). Hence $\left(S_{n}\right)$ is bounded in $L^{\infty}(E)$, and $X_{j}$ is integrable for all $j$. Let $x_{j}=\mathbf{E} X_{j}$; then

$$
\sum_{j=1}^{\infty}\left|\left\langle\infty^{\prime}, \mathbf{E} X_{j}\right\rangle\right|=\sum_{j=1}^{\infty}\left|\mathbf{E}\left\langle x^{\prime}, X_{j}\right\rangle\right| \leqslant \mathbf{E}\left\{\sum_{j=1}^{\infty}\left|\left\langle x^{\prime}, X_{j}\right\rangle\right|\right\} \leqslant K\left(x^{\prime}\right) .
$$

Hence $\sum_{1}^{\infty} x_{j}$ is weakly unconditionally convergent in $E$. Now let $\tilde{X}_{j}=X_{j}-x_{j}$; we then have that $\mathbf{E} \tilde{X}_{j}=0$ and

$$
\begin{aligned}
\sum_{j=1}^{\infty}\left|\left\langle\omega^{\prime}, \tilde{X}_{j}(\omega)\right\rangle\right| & \leqslant \sum_{j=1}^{\infty}\left|\left\langle\infty^{\prime}, X_{j}(\omega)\right\rangle\right|+\sum_{j=1}^{\infty}\left|\left\langle x^{\prime}, x_{j}\right\rangle\right| \\
& \leqslant K\left(x^{\prime}\right)+\sum_{j=1}^{\infty}\left|\left\langle x^{\prime}, X_{j}(\omega)\right\rangle\right| .
\end{aligned}
$$


This shows that $\left(\tilde{X}_{j}\right)$ satisfies $(3.15)$, and so there exists $\Omega_{0} \epsilon \mathscr{F}$, with $P\left(\Omega_{0}\right)=1$ and there exists a constant $K>0$ with

$$
\sum_{j=1}^{\infty}\left|\left\langle x^{\prime}, \tilde{X}_{j}(\omega)\right\rangle\right| \leqslant K\left\|x^{\prime}\right\| \quad \forall \omega \in \Omega_{0}, \forall x^{\prime} \in \mathbb{D}^{\prime} .
$$

It is well known that for a weakly unconditional convergent series $\sum_{1}^{\infty} x_{n}$ we have, for some $L>0$, that

$$
\sum_{j=1}^{\infty}\left|\left\langle x^{\prime}, \infty_{j}\right\rangle\right| \leqslant L\left\|\infty^{\prime}\right\|
$$

Hence

$$
\sum_{j=1}^{\infty}\left|\left\langle x^{\prime}, X_{j}(\omega)\right\rangle\right| \leqslant(K+L)\left\|\alpha^{\prime}\right\| \quad \forall \omega \in \Omega_{0}, \forall x^{\prime} \in \mathbb{B}^{\prime},
$$

and so Theorem 3.6 is proved.

CoroLIART 3.7. Let $\left(X_{1}\right)$ be a sequence of integrable independent $E$-valued random variables such that $\mathbf{E} X_{j}=0$ for all $j \geqslant 1$, and such that the partial sums:

$$
S_{n}=\sum_{j=1}^{n} X_{j}
$$

are bounded in $L^{\infty}(E)$.

If $\mathbb{E}$ does not contain a subspace isomorphic to $c_{0}$, then $\left\{\mathcal{S}_{n}\right\}$ oonverges in $L^{\infty}(E)$.

Proof. By Theorem 3.6 we know that for all $\omega \epsilon \Omega_{0}$, where $P\left(\Omega_{0}\right)=1$, we have that

$$
\sum_{j=1}^{\infty}\left|\left\langle x^{\prime}, X_{j}(\omega)\right\rangle\right| \leqslant K\left\|x^{\prime}\right\| \quad \forall x^{\prime} \in \mathbb{E}^{\prime},
$$

so by Theorem 5 in. [1] we have that $\sum_{1}^{\infty} X_{j}$ converges a.s. and that

$$
\left\|\sum_{1}^{\infty} X_{j}(\omega)\right\| \leqslant K \quad \forall \omega \in \Omega_{0} .
$$

Hence by Theorem 3.5 we have that $\left(S_{n}\right)$ converges in $L^{\infty}(E)$.

4. The comparison principle. Let $\left(X_{j}\right)$ be a sequence of indopendent $E$-valued random variables, and let $\left(\xi_{j}\right)$ and $\left(\eta_{j}\right)$ be sequences of independent scalar valued random variables, we shall then study the series

$$
\sum_{j=1}^{\infty} \eta_{j} X_{j}
$$

in terms of the behaviour of the series

$$
\sum_{j=1}^{\infty} \xi_{j} X_{j}
$$

First we consider the case where $\eta_{j}$ is non-random and $\xi_{j} \equiv 1$.

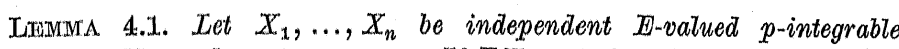
random variables, where $1 \leqslant p<\infty$. If $\mathbf{E} X_{j}=0$ for all $j=1, \ldots, n$, we then have that

$$
\left\{\mathbf{E} \| \sum_{j=1}^{n} a_{j} \cdot X_{j}||^{p}\right\}^{1 / p} \leqslant 2 \max _{1 \in j \leq \leqslant n}\left|a_{j}\right|\left\{\mathbf{E} \| \sum_{j=1}^{n} X_{j}||^{p}\right\}^{1 / p}
$$

for all $a_{1}, \ldots, a_{n} \in \boldsymbol{R}$.

If $X_{j}$ is symmotrio for all $j=1, \ldots, n$, we then have that

$$
\left\{\mathbf{E}\left\|\sum_{j=1}^{n} a_{j} X_{j}\right\|^{p}\right\}^{\alpha / p} \leqslant \max _{1 \leq j \leq n}\left|a_{j}\right|\left\{\mathbf{E}\left\|\sum_{j=1}^{n} X_{j}\right\|^{p}\right\}^{1 / p}
$$

for all $a_{1}, \ldots, a_{n} \in \boldsymbol{R}$.

Proof. If $\sigma \subseteq\{1,2, \ldots, n\}$ it then follows from Theorem 2.6 that

$$
\mathbf{E}\left\|\sum_{j \in \sigma} X_{j}\right\|^{p} \leqslant \mathbf{E}\left\|\sum_{j=1}^{n} X_{j}\right\|^{p} .
$$

If $a_{j}= \pm 1$ for all $j=1, \ldots, n$, we then may put $\sigma=\left\{1 \leqslant j \leqslant n \mid a_{j}=+1\right\}$ and $x=\left\{1 \leqslant j \leqslant n \mid a_{j}=-1\right\}$; we then have

$$
\begin{aligned}
\left\{\mathbf{E}\left\|\sum_{j=1}^{n} a_{j} X_{j}\right\|^{p}\right\}^{1 / p} & \leqslant\left\{\mathbf{E}\left\|\sum_{j \in \sigma} X_{j}\right\|^{p}\right\}^{1 / p}+\left\{\mathbf{E}\left\|\sum_{j \in \boldsymbol{x}} X_{j}\right\|^{p}\right\}^{1 / p} \\
& \leqslant 2\left\{\mathbf{E}\left\|\sum_{j=1}^{n} X_{j}\right\|^{p}\right\}^{1 / p}
\end{aligned}
$$

If $K_{n}=\left\{\left(a_{1}, \ldots, a_{n}\right) \in \ddot{\boldsymbol{R}}^{n}|| a_{1}|\leqslant 1, \ldots,| a_{n} \mid \leqslant 1\right\}$, then $K_{n}$ is a compact convex subset of $\boldsymbol{R}^{n}$, whose extreme points are the $2^{n}$ points: ( $\pm 1, \pm 1, \ldots$ $\ldots, \pm 1)$. Thus if $a=\left(a_{1}, \ldots, a_{n}\right) \in K_{n}$ then by Caratheodory's theorem $a$ is a convex combination of at most $n+1$ extreme points. That is, we can find $\lambda_{m} \geqslant 0$ for $m=1, \ldots, n+1$ with

$$
a_{j}=\sum_{m=1}^{n+1} \lambda_{m} a_{j m}, \quad \sum_{m=1}^{n+1} \lambda_{m}=1
$$


where $a_{j m}= \pm 1$. Hence we find

$$
\begin{aligned}
\left\{\mathbf{E}\left\|\sum_{j=1}^{n} a_{j} X_{j}\right\|^{p}\right\}^{1 / p} & =\left\{\mathbf{E}\left\|\sum_{m=1}^{n+1} \lambda_{m}\left(\sum_{j=1}^{n} a_{j m} X_{j}\right)\right\|^{p}\right\}^{1 / p} \\
& \leqslant \sum_{m=1}^{n+1} \lambda_{m}\left\{\mathbf{E}\left\|\sum_{j=1}^{n} a_{j m} X_{j}\right\|^{p}\right\}^{1 / p} \\
& \leqslant 2\left\{\mathbf{E}\left\|\sum_{j=1}^{n} X_{j}\right\|^{p}\right\}^{1 / p}
\end{aligned}
$$

from which the first part of the lemma follows easily.

If $X_{j}$ is symmetric for all $j=1, \ldots, n$, then we have

$$
\mathbf{E}\left\|\sum_{j=1}^{n} a_{j} X_{j}\right\|^{p}=\mathbf{E}\left\|\sum_{j=1}^{n} X_{j}\right\|^{p}
$$

whenever $a_{j}= \pm 1$. The last part of the lemma follows as above.

CorolLaRY 4.2. Let $X_{1}, \ldots, X_{n}$ be $\mathbb{E}$-valued, independent, $p$-integrable random variables with $1 \leqslant p<\infty$ amd $\mathbf{E} X_{j}=0$ for all $j=1, \ldots, n$. If $\eta_{1}, \ldots, \eta_{n}$ are real valued, $p$-integrable random variables, so that $\left(X_{1}, \ldots, X_{n}\right)$ and $\left(\eta_{1}, \ldots, \eta_{n}\right)$ are independent, then

$$
2^{-p} \mathbf{E}\left(\min _{1 \leqslant j \leqslant n}\left|\eta_{j}\right|^{p}\right) \mathbf{E}\left\|\sum_{j=1}^{n} X_{j}\right\|^{p} \leqslant \mathbf{E}\left\|\left.\sum_{j=1}^{n} \eta_{j} X_{j}\right|^{p} \leqslant 2^{p} \mathbf{E}\left(\max _{1<j<\leqslant \infty}\left|\eta_{j}\right|^{p}\right) \mathbf{E}\right\| \sum_{j=1}^{n} X_{j} \|^{p} .
$$

Proof. From Lemma 4.1 it follows that

$$
2^{-p} \min \left|\boldsymbol{a}_{j}\right|^{p} \mathbf{E}\left\|\sum_{j=1}^{n} X_{j}\right\|^{p} \leqslant \mathbf{E}\left\|\sum_{j=1}^{n} a_{j} X_{j}\right\|^{p} \leqslant 2^{p} \max \left|a_{j}\right|^{p} \mathbf{E}\left\|\sum_{j=1}^{n} X_{j}\right\|^{p}
$$

for all $a_{1}, \ldots, a_{n}$ (the first inequality follows from Lemma 4.1 by putting $X_{j}=a_{j} X_{j}$ and $a_{j}=1 / a_{j}$ ). So the corollary follows by integrating with respect to the distribution law of $\left(\eta_{1}, \ldots, \eta_{n}\right)$.

THEOREM 4.3. Let $\left(\eta_{j}\right)$ and $\left(\xi_{j}\right)$ be sequences of independent, real valued, $p$-integrable random variables with $1 \leqslant p<\infty$, and let $\left(X_{j}\right)$ be a sequence of $E$-valued, independent p-integrable random variables such that

(4.3) $\left(X_{j}\right)$ and $\left(\xi_{j}\right)$ are independent, and $\left(X_{j}\right)$ and $\left(\eta_{j}\right)$ are independent,

$$
\mathbf{E}\left(\eta_{j} X_{j}\right)=\mathbf{E}\left(\xi_{j} X_{j}\right)=0 \quad \forall j \geqslant 1 \text {. }
$$

Then we have

$$
\mathbf{E}\left\|\sum_{j=1}^{n} \eta_{j} X_{j}\right\|^{p} \leqslant\left(\frac{8}{a}\right)^{p} \mathbf{E}\left(N^{p}\right) \mathbf{E}\left\|\sum_{j=1}^{n} \xi_{j} X_{j}\right\|^{p} \quad \forall n .
$$

Remark. In. my original version I assumed that $P\left(\underset{j}{\inf }\left|\xi_{j}\right|>0\right)>0$ instead of (4.2). The strengthening of the theorem is based on an idea of B. Maurey (oral communication).

Proof. Let $\left(\varepsilon_{j}\right)$ be a Bernoulli sequence, which is independent of $\left(\left(X_{j}\right),\left(\xi_{j}\right),\left(\eta_{j}\right)\right)$. By Oorollary 4.2 , with $X_{j}=\eta_{j} X_{j}$ and $\eta_{j}=\varepsilon_{j}$, we have

$$
\mathbf{E}\left\|\sum_{j=1}^{n} \eta_{j} X_{j}\right\|^{p} \leqslant 2^{p} \mathbf{E}\left\|\sum_{j=1}^{n} \eta_{j} z_{j} X_{j}\right\|^{p}
$$

Since $\varepsilon_{j} X_{j}$ is symmetric and $\left(\eta_{j}\right)$ is independent of $\left(\varepsilon_{j} X_{j}\right)$, we find by Corollary 4.2 that

$$
\mathbf{E}\left\|\sum_{j=1}^{n} \eta_{j} X_{j}\right\|\left\|^{p} \leqslant 2^{p} \mathbf{E}\right\| \sum_{j=1}^{n} \eta_{j} \varepsilon_{j} X_{j}\left\|^{p} \leqslant 4^{p} \mathbf{E}\left(N^{p}\right) \mathbf{E}\right\| \sum_{j=1}^{n} \varepsilon_{j} X_{j} \|^{p} .
$$

Let $a_{j}=\mathbf{E}\left|\xi_{j}\right| ;$ we then have by Lemma 4.1

$$
\begin{aligned}
\mathbf{E}\left\|\sum_{j=1}^{n} \eta_{j} X_{j}\right\|^{\boldsymbol{p}} & \leqslant 4^{p} \mathbf{E}\left(\mathcal{N}^{p}\right) \max _{1 \leqslant j \leqslant n}\left(\frac{1}{a_{j}}\right)^{p} \mathbf{E}\left\|\sum_{j=1}^{n} a_{j} \varepsilon_{j} X_{j}\right\|^{p} . \\
& \leqslant\left(\frac{4}{a}\right)^{p} \mathbf{E}\left(N^{p}\right) \mathbf{E}\left\|\sum_{j=1}^{n} a_{j} \varepsilon_{j} X_{j}\right\|^{\boldsymbol{p}} .
\end{aligned}
$$

Let $\mathscr{F}$ be the $\sigma$-algebra generated by $\left(\varepsilon_{1}, \ldots, \varepsilon_{n}, X_{1}, \ldots, X_{n}\right)$, and define

$$
\varepsilon_{j}^{*}=\left\{\begin{array}{rll}
-\varepsilon_{j} & \text { if } \quad \xi_{j}<0, \\
\varepsilon_{j} & \text { if } \quad \xi_{j} \geqslant 0 .
\end{array}\right.
$$

Then we have

$$
\begin{aligned}
\left\|\sum_{j=1}^{n} a_{j} \varepsilon_{j}, X_{j}\right\|^{p}=\left\|\mathbf{E}\left(\sum_{j=1}^{n}\left|\xi_{j}\right| \varepsilon_{j} X_{j} \mid \mathscr{F}\right)\right\|^{p} & \leqslant \mathbf{E}\left(\left\|\sum_{j=1}^{n}\left|\xi_{j}\right| \varepsilon_{j} X_{j}\right\|^{p} \mid \mathscr{F}\right) \\
& =\mathbf{E}\left(\left\|\sum_{j=1}^{n} \xi_{j} \varepsilon_{j}^{*} X_{j}\right\|^{p} \mid \mathscr{F}\right) .
\end{aligned}
$$

and so we find

$$
\mathbf{E}\left\|\sum_{j=1}^{n} \eta_{j} X_{j}\right\|^{p} \leqslant\left(\frac{4}{a}\right)^{p} \mathbf{E}\left(N^{j}\right) \mathbf{E}\left\|\sum_{j=1}^{n} \varepsilon_{j}^{*} \xi_{j} X_{j}\right\|^{p}
$$


It is easily checked that $\left(\varepsilon_{j}^{*}\right)$ and $\left(\left(\xi_{j}\right),\left(X_{j}\right)\right)$ are independent, and so by Corollary 4.2 we find

$$
\mathbf{E}\left\|\sum_{j=1}^{n} \eta_{j} X_{j}\right\|^{p} \leqslant\left(\frac{8}{a}\right)^{p} \mathbf{E}\left(N^{p}\right) \mathbf{E}\left\|\sum_{j=1}^{n} \xi_{j} X_{j}\right\|^{p}
$$

CoRoLiary 4.4. Let $\left(\xi_{j}\right),\left(\eta_{j}\right)$ and $\left(X_{j}\right)$ be a sequence of real (respectively $E$-valued), independent $p$-integrable random variables satisfying (4.1)(4.4). If

\section{we then have}

$$
S_{n}=\sum_{j=1}^{n} \xi_{j} X_{j}, \quad T_{n}=\sum_{j=1}^{n} \eta_{j} X_{j}
$$

(4.5) If $\left(S_{n}\right)$ is bounded in $L^{p}(E)$, then so is $\left(T_{n}\right)$.

(4.6) If $\left(S_{n}\right)$ converges in $L^{p}(E)$, then so does $\left(T_{n}\right)$.

(4.7) If $\left(S_{n}\right)$ is bounded in $L^{p}(E)$, and if $\eta_{j} \underset{j \rightarrow \infty}{\longrightarrow} 0$ a.s., then $\left(T_{n}\right)$ converges in $L^{p}(E)$.

Proof. Immediate consequence of Theorem 4.3.

5. Boundedness and convergence. It is well known that if $E=\boldsymbol{R}$ (or even if $E$ is a Hilbert space) then boundedness of the partial sums

$$
S_{n}=\sum_{j=1}^{n} X_{j}
$$

where $\left(X_{j}\right)$ is a symmetric sequence, implies a.s. convergence of $\left(S_{n}\right)$. Now from Proposition 2.8 it follows that a Banach space $E$ has this property if and only if

$$
B(E)=O(E) \text {. }
$$

We shall in this section study the class of Banach spaces with this property.

THEOREM 5.1. If $(\Omega, \mathscr{F}, P)$ is a probability space on which we can define a Bernouilli sequence then the following statements are equivalent:

$$
\begin{array}{ll}
(5.1) & B(E) \neq c_{0}(E), \\
(5.2) & B(E) \neq O(E),
\end{array}
$$

(5.3) $\exists 1 \leqslant p<\infty$, such that $L^{p}(\Omega, \mathscr{F}, P, E)$ contains a closed subspace isomorphic to $c_{0}$,

(5.4) $\forall 1 \leqslant p<\infty, L^{p}(\Omega, \mathscr{F}, P, \mathbb{E})$ contains a closed subspace isomorphio to $c_{0}$.

Remark. I conjecture that (5.1) $-(5.4)$ are equivalent to

(5.5) $E$ contains a closed subspace isomorphio to $c_{0}$.
Notice that (5.5) implies (5.2), as $\left(e_{n}\right) \epsilon B(E)$, but $\left(e_{n}\right) \notin c_{0}(E)$ if $e_{n}$ is the $n$th unit vector in $c_{0}$. Notice also that Corollary 3.7 gives a partial answer to the converse implication.

Pro of of Theorem 5.1. (5.4) implies (5.3) for trivial reasons. Suppose that (5.3) holds, and let $\left(\varepsilon_{j}\right)$ be a Bernoulli sequence defined on $(\Omega, \mathscr{F}, P)$. By assumption there exist a $1 \leqslant p<\infty$ and $f_{j} \in I^{p}(E)$ such that

$$
\begin{aligned}
& a \leqslant\left\|f_{j}\right\|_{p} \leqslant b \quad \forall j \geqslant 1, \quad \text { where } 0<a \leqslant b<\infty, \\
& \left\|\sum_{j=1}^{n} \alpha_{j} f_{j}\right\|_{p} \leqslant K \max _{1 \leqslant j \leqslant n}\left|\alpha_{j}\right| \quad \forall n \geqslant 1, \forall a_{1}, \ldots, \alpha_{n} \in \boldsymbol{R}
\end{aligned}
$$

where $K$ is a finite constant. On the probability space $(\Omega \times \Omega, \mathscr{F} \otimes \mathscr{F}$, $P \times P)=\left(\Omega^{\prime}, \mathscr{F}^{\prime}, P^{\prime}\right)$ we define

$$
f_{j}^{\prime}\left(\omega_{1}, \omega_{2}\right)=\varepsilon_{j}\left(\omega_{1}\right) f_{j}\left(\omega_{2}\right) \quad \text { for } \quad\left(\omega_{1}, \omega_{2}\right) \in \Omega^{\prime} .
$$

It is then easily seen that

$$
\begin{gathered}
a \leqslant\left\|f_{j}^{\prime}\right\|_{p} \leqslant b \quad \forall j \geqslant 1, \\
\left\|\sum_{j=1}^{n} \alpha_{j} f_{j}^{\prime}\right\|_{p} \leqslant K \max _{1 \leqslant j \leqslant n}\left|a_{j}\right| \quad \forall n \geqslant 1, \forall \alpha_{1}, \ldots, \alpha_{n} \in \boldsymbol{R},
\end{gathered}
$$

and that $\left(f_{j}^{\prime}\right)$ is a symmetric sequence. From (5.7) it follows that $g_{n}^{\prime}=\sum_{j=1}^{n} f_{j}^{\prime}$ s bounded in $L^{p}(E)$. So by Proposition 2.8 we have that $\left(f_{j}^{\prime}\left(\omega^{\prime}\right)\right) \in B(E)$ for a.a. $\omega^{\prime} \in \Omega^{\prime}$. Now if $\left(f_{j}^{\prime}\left(\omega^{\prime}\right)\right) \in C(E)$ a.s., we then know that $\left(g_{n}^{\prime}\right)$ converges a.s. to a random variable $g^{\prime}$ by Proposition 2.8. So by Fatou's lemma we have

i.e. $g^{\prime} \epsilon L^{p}(E)$. Since

$$
\mathbf{E}\left\|g^{\prime}\right\|^{p} \leqslant \liminf _{n \leqslant \infty} \mathbf{E}\left\|g_{n}^{\prime}\right\|^{p} \leqslant K^{p}
$$

$$
P\left(\sup _{n}\left\|g_{r}^{\prime}\right\| \geqslant t\right) \leqslant 2 P\left(\left\|g^{\prime}\right\| \geqslant t\right),
$$

we have that $\sup _{n}\left\|g_{n}^{\prime}\right\| \epsilon L^{p}(\boldsymbol{R})$. Thus by Lebesgue's theorem of dominated convergence we have that $\left(g_{x}^{\prime}\right)$ converges in $L^{p}(E)$, but this contradicts the fact that $\left\|f_{j}^{\prime}\right\|_{p} \geqslant a$ for all $j \geqslant 1$. Hence we have

$$
\begin{gathered}
\left(f_{j}^{\prime}\left(\omega^{\prime}\right)\right) \in B(E) \text { for a.a. } \omega^{\prime} \epsilon \dot{\Omega}^{\prime}, \\
\left(f_{j}^{\prime}\left(\omega^{\prime}\right)\right) \notin C(E) \text { for all } \omega^{\prime} \in A^{\prime} \text { where } P\left(A^{\prime}\right)>0 .
\end{gathered}
$$

That is, $B(E) \neq \dot{C}(E)$. And so (5.3) implies (5.2). 
Now suppose that $B(E) \neq O(E)$. And let $\left(x_{j}\right)$ be ar sequence in $B(E) \backslash O(E)$. Since $\sum_{1}^{n} \varepsilon_{j} x_{j}$ is not a Cauchy sequence in $L^{1}(E)$, we can find $a>0$ and a sequence $1=n_{1}<n_{2}<\ldots$ such that

Now let

$$
\mathbf{E}\left\|\sum_{n_{k} \leqslant j<n_{k+1}} \varepsilon_{j} x_{j}\right\| \geqslant a \quad \forall k=1,2, \ldots
$$

$$
X_{k}=\sum_{n_{k} \leqslant j<n_{k+1}} \varepsilon_{j} x_{j} \quad \text { for } \quad k=1,2, \ldots
$$

Then $X_{1}, X_{2}, \ldots$ are independent symmetric random variables. Furthermore, if $M(\omega)=\sup _{n}\left\|\sum_{1}^{n} \varepsilon_{j}(\omega) x_{j}\right\|$, then by Theorem 3.1 we have

$$
\begin{gathered}
M(\omega)<\infty \text { a.s. and } \mathbf{E} M<\infty, \\
\mathbf{E}\left\|\sum_{v=1}^{k} X_{v}\right\|=\mathbf{E}\left\|_{1 \leqslant j<n_{t+1}} \varepsilon_{j} x_{j}\right\| \leqslant \mathbf{E} M<\infty, \\
\mathbf{E}\left\|X_{v}\right\| \geqslant a \quad \forall v \geqslant 1, \\
\left\|X_{v}(\omega)\right\| \leqslant 2 M(\omega) \quad \forall v \geqslant 1 .
\end{gathered}
$$

This implies that $P\left(X_{v} \rightarrow 0\right)>0$. And from Proposition 2.8 it follows that $\left(X_{v}(\omega)\right) \in B(E)$ a.s. Hence $B(E) \$ c_{0}(E)$. That is, (5.2) implies (5.1).

Now suppose that $B(E) \notin c_{0}(E)$. Then we can find $\left(x_{j}\right) \in B(E)$ such that $\left\|x_{j}\right\|=1$ for all $j=1,2, \ldots$ Now let $\left(\varepsilon_{j}\right)$ be a Bernoulli sequence defined on $(\Omega, \mathscr{F}, P)$ and put

$$
X_{j}=\varepsilon_{j} x_{j} .
$$

Then by Lemma 4.1 and Theorem 2.6 we have

$$
\left|a_{j}\right|=\left(\mathbf{E}\left\|a_{j} X_{j}\right\|^{p}\right)^{1 / p} \leqslant\left(\mathbf{E}\left\|\sum_{v=1}^{n} a_{v} X_{v}\right\|^{p}\right)^{1 / p} \leqslant \max _{1 \leqslant v \leqslant n}\left|a_{v}\right|\left(\mathbf{E}\left\|\sum_{v=1}^{n} \varepsilon_{v} x_{v}\right\|^{p}\right)^{1 / p}
$$

for $n \geqslant j \geqslant 1$ and all $a_{1}, \ldots, a_{n} \in \boldsymbol{R}$. Hence if

$$
K=\sup _{n}\left(\mathbf{E}\left\|\sum_{v=1}^{n} \varepsilon_{v} x_{v}\right\|^{p}\right)^{1 / p}
$$

then

$$
\max _{1 \leqslant j \leqslant n}\left|a_{j}\right| \leqslant\left\|\sum_{j=1}^{n} a_{j} X_{j}\right\|^{p} \leqslant K \max _{1 \leqslant j \leqslant n}\left|a_{j}\right|
$$

And by Corollary 4.4 we have that $\sum_{i}^{\infty} a_{j} X_{j}$ exist for all $\left(a_{j}\right) \epsilon c_{0}$. Then the inequalities above show that the map

$$
\left(a_{j}\right) \curvearrowright \sum_{1}^{\infty} a_{j} X_{j}
$$

is an isomorphism of $c_{0}$ into a closed subspace of $L^{p}(E)$ for all $p \in[1, \infty)$. That is, (5.1) implies $(5,4)$. and so the theorem is proved.

ThDOREM 5.2. If $E$ has the Radon-Nikodym property, then $B(E)$ $=O(E)$.

Remark. Later we shall see that if $E=L^{p}(S, \Sigma, \mu)$ for some measure space $(S, \Sigma, \mu)$ and some $1 \leqslant p<\infty$, then $B(E)=O(E)$. Now it is well known that $L^{1}[0,1]$ does not have the Radon-Nikodym property. Thus $B(E)=C(E)$ does not imply the Radon-Nikodym property.

Proof of Theorem 5.2. Immediate consequence of Theorem 6 in $[2]$.

We shall now show that if the partial sums, $S_{n}=\sum_{1}^{n} X_{j}$, are a.s. bounded and relatively compact, then the series $\sum_{1}^{\infty} X_{j}$ converges a.s. Before we state the theorem, we observe the following application of Chatterji's results on vector-valued martingales (see [2]; compare also Theorem 4.1 in [8]):

THeOREM 5.3. Let $\left(X_{n}\right)$ be independent integrable E-valued random variables, and let $F^{\prime}$ be a subset of $E^{\prime}$ such that $F^{\prime}$ separates points in $E$. If there exists $S \in L^{1}(E)$ such that

$$
\left\langle x^{\prime}, \sum_{j=1}^{n} X_{j}\right\rangle_{n \rightarrow \infty}\left\langle x^{\prime}, S\right\rangle \text { in probability }
$$

for all $x^{\prime} \in F^{\prime}$, and $\mathbf{E} X_{j}=0$ for all $j \geqslant 1$, then the series

$$
\sum_{1}^{\infty} X_{j}
$$

converges to $S$ a.s. and in $L^{1}(E)$,

Pro of. By Corollary 3.3 we have that $\left\langle x^{\prime}, S_{n}\right\rangle \underset{n \rightarrow \infty}{\longrightarrow}\left\langle x^{\prime}, S\right\rangle$ in $L^{1}(\boldsymbol{R})$ for all $x^{\prime} \in F^{\prime}$, where $S_{n}$ is the partial sum

$$
S_{n}=\sum_{j=1}^{n} X_{j}
$$

Hence $\mathbf{E S}=0$ as $\boldsymbol{F}^{\prime}$ separates $E$. 
Now let $\mathscr{F}_{n}$ be the $\sigma$-algebra spanned by $\left\{X_{1}, \ldots, X_{n}\right\}$; then for $x^{\prime} \in H^{\prime}$ we have

$$
\int_{A}\left\langle x^{\prime}, S_{n}\right\rangle d P=\int_{A}\left\langle x^{\prime}, S\right\rangle d P \quad \forall A \in \mathscr{F}_{n}
$$

since $\left\langle x^{\prime}, S-S_{n}\right\rangle=\sum_{j=n+1}^{\infty}\left\langle x^{\prime}, X_{j}\right\rangle$ is independent of $\mathscr{F}_{n}$ and has mean 0 .

Now since $S_{n}$ and $S$ both belong to $L^{1}(E)$ and $F^{\prime}$ separates points of $E$, we have that

$$
\int_{A} S_{n} d P=\int_{A} S d P \quad \forall A \in \mathscr{F}_{n}
$$

That is, $S_{n}=\mathbf{E}\left(S \mid \mathscr{F}_{n}\right)$. Thus by Theorem 1 (a) in [2] we have that $\left(S_{n}\right)$ converges in $L^{1}(E)$, and so by Theorem 2.5, $\left(S_{n}\right)$ converges a.s. Also the limit of $\left(S_{n}\right)$ is of course equal to $S$ as $F^{\prime}$ separates points of $E$.

THEOREM 5.4. Let $\tau$ be a locally convex Hausdorff topology on $\mathbb{E}$ which is weaker than the norm topology, and such that the closed unit ball in $E$ is $\tau$-closed. Let $x=\left(x_{j}\right) \in E^{\infty}$, and put

$$
F(\omega)=\left\{\sum_{j=1}^{n} \varepsilon_{j}(\omega) x_{j} \mid n \geqslant 1\right\} \quad \text { for } \quad \omega \in \Omega,
$$

where $\left(\varepsilon_{j}\right)$ is a Bernoulli sequence.

If $E$ is separable and $F(\omega)$ is relatively $\tau$-compact for a.a. $\omega$, then $x \in O(E)$.

Proof. Let us put $S_{n}=\sum_{1}^{n} \varepsilon_{j} x_{j}$, and let $F^{\prime}$ be the dual of $(E, \tau)$. Then $F^{\prime} \subseteq E^{\prime}$ as $\tau$ is weaker than $\|\cdot\|$.

Now, as $E$ is separable, we can find a countable set $T \subseteq Z^{\prime}$ such that $T$ separates $E$.

As $\left(\left\langle x^{\prime} x_{n}\right\rangle\right) \in B(\boldsymbol{R})=C(\boldsymbol{R})$, we have that

$$
\Phi\left(x^{\prime}, \omega\right)=\lim _{n \rightarrow \infty}\left\langle x^{\prime}, S_{n}(\omega)\right\rangle
$$

exists for all $x^{\prime} \epsilon T$ and all $\omega \notin N_{0}$ where $P\left(N_{0}\right)=0$. By assumption there exists a null set $N_{1} \supseteq N_{0}$ such that $\left\{S_{n}(\omega) \mid n \geqslant 1\right\}$ is relatively $\tau$-compact for all $\omega \notin N_{1}$, and so if $\omega \notin N_{1}$ we can find a sequence (depending on $\omega$ ): $n_{1}<n_{2}<\ldots$ such that for some $S(\omega) \in E$ we have $\tau$ - $\lim _{j \rightarrow \infty} S_{n_{j}}(\omega)=S(\omega)$. Since $T \subseteq F^{\prime}$, we find that

$$
\left\langle x^{\prime}, S(\omega)\right\rangle=\lim _{j \rightarrow \infty}\left\langle x^{\prime}, S_{n_{j}}(\omega)\right\rangle=\Phi\left(x^{\prime}, \omega\right)
$$

for all $x^{\prime} \in T$. This shows that for $\omega \notin N_{1}$ we have that $\left\{S_{n}(\omega)\right\}$ has one and only one $\tau$-limit point as $T$ separates $E$. That is,

$$
S(\omega)=\tau-\lim _{n \rightarrow \infty} S_{n}(\omega)
$$

exists for all $\omega \notin N_{1}$.

Since $(E,\|\cdot\|)$ is a Polish space, we have that $(E, \tau)$ is a standard space, and the Borel sets of $(E, \tau)$ are equal to Borel sets of $(E,\|\cdot\|)$ (see, for example, Proposition III.1.7 and Corollary III.2.6 in [6]). Hence, by Theorem IV.2.4 in [6], we have that $S$ is an $E$-valued random variable. The property that the unit ball is $\tau$-closed is easily seen to be equivalent to

$$
\|x\|=\sup \left\{\left|\left\langle x^{\prime}, x\right\rangle\right|: x^{\prime} \in F^{\prime},\left\|x^{\prime}\right\| \leqslant 1\right\} \quad \forall x \in E .
$$

Now since $E$ is separable, we can find a countable set $B^{\prime} \subseteq\left\{x^{\prime} \in F^{\prime} \mid\left\|x^{\prime}\right\|\right.$ $\leqslant 1\}$, with the property

$$
\|x\|=\sup \left\{\left|\left\langle x^{\prime}, x\right\rangle\right|: x^{\prime} \in B^{\prime}\right\} \quad \forall x \in E .
$$

Now let $B^{\prime}=\left\{x_{1}^{\prime}, x_{2}^{\prime}, \ldots\right\}$, and define

$$
\begin{aligned}
S_{j}^{k} & =\sum_{v=1}^{j} \varepsilon_{j}\left\langle x_{k}^{\prime}, x_{v}\right\rangle=\left\langle x_{k}^{\prime}, S_{j}\right\rangle, \\
S^{k} & =\sum_{v=1}^{\infty} \varepsilon_{j}\left\langle x_{k}^{\prime}, x_{v}\right\rangle=\left\langle x_{k}^{\prime}, S\right\rangle, \\
\tilde{S}_{j}^{k} & =\sum_{v=1}^{j} \varepsilon_{j}\left\langle x_{k}^{\prime}, x_{v}\right\rangle-\sum_{v=j+1}^{\infty} \varepsilon_{j}\left\langle x_{k}^{\prime}, x_{v}\right\rangle \\
& =2 S_{j}^{k}-S^{k} .
\end{aligned}
$$

(Notice that the sums converge a.s. since $x_{n}^{\prime}$ is $\tau$-continuous.) Now let $t>0$, and define

$$
T=\inf \left\{j \mid\left\|S_{j}\right\|>t\right\} \quad(\inf (\varnothing)=\infty) .
$$

We then have

$$
P\left(\sup _{j}\left\|S_{j}\right\|>t\right)=P(T<\infty)=\sum_{j=1}^{\infty} P(T=j) .
$$

If $T=j$ we then have, for some $k \geqslant 1$, that $\left|S_{j}^{\prime c}\right|>t$, and since $S_{j}^{h}=\frac{1}{2} \tilde{S}_{j}^{h}+$ $+\frac{1}{2} S^{\text {to }}$ we have that either

$$
\sup \left|S^{k}\right|>t
$$

or 
Hence we have

$$
P\left(\sup _{j}\left\|S_{j}\right\|>t\right) \leqslant \sum_{j=1}^{\infty}\left\{P\left(T=j, \sup _{k}\left|\tilde{S}_{j}^{k}\right|>t\right)+P(T=j,\|S\|>t)\right\} .
$$

Since $\varepsilon_{j}$ is symmetric for all $j$, we have that the two families, $S_{1}, \ldots, S_{j}$, $S^{1}, S^{2}, \ldots$ and $S_{1}, \ldots, S_{j}, \tilde{S_{j}^{1}}, \tilde{S}_{j}^{2}, \ldots$ are equidistributed for all $j=1, \ldots$, hence

$$
P\left(T=j, \sup _{k}\left|\tilde{S}_{j}^{k}\right|>t\right)=P(T=j,\|S\|>t)
$$

and so

$$
P\left(\sup _{j}\left\|S_{j}\right\|>t\right) \leqslant 2 P(\|S\|>t) \quad \forall t>0 .
$$

Hence $\sup _{j}\left\|S_{j}\right\|<\infty$ a.s., and so $\left(x_{j}\right) \in B(E)$, and $M=\sup _{j}\left\|S_{j}\right\|$ is integrable. Now, since the unit ball is $\tau$-closed, we have that $\|S\| \leqslant M$, and so $S \in L^{1}(E)$, and the theorem then follows from Theorem 5.3.

COROLLARY 5.5. Let $\tau$ be a locally convex Hausdorff topology on $\mathbb{E}$ such that $\tau$ is weaker than the norm topology, and the unit ball of $E$ is $\tau$-closed. Let $\left(X_{n}\right)$ be a symmetric sequence of $\mathbb{E}$-valued random variables and put

$$
F(\omega)=\left\{\sum_{j=1}^{n} X_{j}(\omega) \mid n \geqslant 1\right\}
$$

If $E$ is separable and $F(\omega)$ is relatively $\tau$-compact for almost all $\omega \in \Omega$, then $\sum_{1}^{\infty} X_{j}$ converges a.s.

CoRollary 5.6. Let $S$ be a compact metric space and let $X_{0}(t, \omega)$, $X_{1}(t, \omega), X_{2}(t, \omega), \ldots$ be stochastic processes with time set $S$. Suppose that (5.10) $\quad X_{n}(t, \cdot)$ is symmetric for all $t \in S$, and $n \geqslant 0$;

(5.11) $X_{n}(\cdot, \omega)$ is continuous and real for almost all $\omega \epsilon \Omega$, and all $n \geqslant 0$;

(5.12) The processes $\left\{X_{n}(t) \mid t \epsilon S\right\}$ for $n=1,2, \ldots$ are independent;

(5.13) $\quad X_{0}(t)=\sum_{n=1}^{\infty} X_{n}(t) \quad$ a.s. for all $t \in S$.

Then the series $\sum_{1}^{\infty} X_{n}(t, \omega)$ converges uniformly in $t \in S$ for almost all $\omega \in \Omega$.

6. Weak convergence. If $\left(X_{n}\right)$ is a sequence of $E$-valued random variables and $\tau$ is a topology on $E$ that is weaker than the norm-topology, we say that $X_{n}$ converges $\tau$-veakly to $\mu$, where $\mu$ is a probability measure on $(E, \mathscr{B}(E))$, if we have

$$
\lim _{n \rightarrow \infty} \mathbf{E} f\left(X_{n}\right)=\int_{E} f(x) \mu(d x)
$$

for all $\tau$-continuous, bounded functions $f$ from $E$ into $\boldsymbol{R}$. And we say that $\left(X_{n}\right)$ is $\tau$-tight if for all $\varepsilon>0$ there exists a $\tau$-compact set $K \subseteq E$ with

$$
P\left(X_{n} \notin K\right)<\varepsilon \quad \forall n \geqslant 1 .
$$

LEMma 6.1. Let $\Phi$ be a map from $F^{\prime} \times \Omega$ into $\boldsymbol{R}$, where $F^{\prime}$ is a linear subspace of $E^{\prime}$. Suppose that $E$ is separable and $\mu$ is a probabitity measure on $(E, \mathscr{B}(E))$ such that

(6.1) $\Phi\left(x^{\prime}, \cdot\right)$ is measurable for all $x^{\prime} \in F^{\prime}$;

(6.2) $P\left(\Phi\left(x^{\prime}\right) \in A\right)=\mu\left(x \in E \mid\left\langle x^{\prime}, x\right\rangle \in A\right) \quad \forall x^{\prime} \in F, \forall A \in \mathscr{B}(\boldsymbol{R})$.

Then there exists an $E$-valued random variable $X$ such that for $x^{\prime} \in F^{\prime}$ we have

$$
\Phi\left(x^{\prime}, \omega\right)=\left\langle x^{\prime}, X(\omega)\right\rangle \text { for a.a. } \omega \in \Omega .
$$

Proof. Since $E$ is separable, there exists a countable subset $T^{\prime} \subseteq T^{\prime}$ such that $T^{\prime}$ is sequentially $\sigma\left(E^{\prime}, E\right)$-dense in $F^{\prime}$ (i.e., for all $x^{\prime} \in F^{\prime}$ exists a sequence $\left(t_{n}^{\prime}\right) \subseteq T^{\prime}$ with $t_{n}^{\prime} \underset{n \rightarrow \infty}{\longrightarrow} x^{\prime}$ in $\left.\sigma\left(E^{\prime}, E\right)\right)$.

Now let $T^{\prime}=\left\{x_{1}^{\prime}, x_{2}^{\prime}, \ldots\right\}$ be an enumeration of $T$ and define

$$
\Psi(\omega)=\left(\Phi\left(x_{j}^{\prime}, \omega\right)\right)_{j=1}^{\infty} .
$$

Then $\Psi(\cdot)$ is a measurable map from $\Omega$ into $\boldsymbol{R}^{\infty}$. Distribution of $\Psi$ is given by

$$
P_{\mathbf{\Psi}}(A)=\mu\left(x \mid\left(\left\langle x_{j}^{\prime}, x\right\rangle\right)_{j=1}^{\infty} \epsilon A\right) \cdot \forall A \in \mathscr{B}\left(\boldsymbol{R}^{\infty}\right) .
$$

Now let $R_{0} \subseteq \boldsymbol{R}^{\infty}$ be the set

$$
R_{0}=\left\{\left(t_{j}\right) \in \boldsymbol{R}^{\infty} \mid \exists x \in E \text { with } t_{j}=\left\langle x_{j}^{\prime}, x\right\rangle \forall j \geqslant 1\right\} .
$$

Since $R_{0}$ is a continuous image of $E$, we have that $R_{0}$ is an analytic space in the sense of [6] Chapter III.1, and obviously $P_{\Psi}\left(R_{0}\right)=1$. (Notice that $R_{0}$ is universally measurable.) Now consider the set

$$
A=\left\{(t, x) \in R_{0} \times E \mid t_{j}=\left\langle x_{j}^{\prime}, x\right\rangle \forall j \geqslant 1\right\} .
$$

$A$ is then a closed subset of $R_{0} \times E$, and so $A$ is analytic. By definition of $R_{0}$ we know that the projection of $A$ onto $R_{0}$ is equal to $R_{0}$. Thus by Theorem III.9.6 of [6] exists a universally measurable map $\varphi: R_{0} \curvearrowright E$ whose graph is contained in $A$, i.e.

$$
\left\langle x_{j}^{\prime}, \varphi(t)\right\rangle=t_{j} \quad \forall t=\left(t_{j}\right) \in R_{0} .
$$

Now let us define

$$
X(\omega)=\left\{\begin{array}{lll}
p(\Psi(\omega)) & \text { if } & \omega \epsilon \Psi^{-1}\left(R_{0}\right) \\
0 & \text { if } & \omega \in \Psi^{-1}\left(R_{0}\right) .
\end{array}\right.
$$


Then $X$ is a $P$-measurable map from $\Omega$ into $E$, and as $E$ is separable we have that $X$ is an $E$-valued random variable.

If $\omega \in \Psi^{-1}\left(R_{0}\right)$ we then have

$$
\left\langle x^{\prime}, X(\omega)\right\rangle=\left\langle x^{\prime}, \varphi(\Psi(\omega))\right\rangle=\Phi\left(x^{\prime}, \omega\right) \quad \forall x^{\prime} \in T^{\prime},
$$

and since $P\left(\Psi^{-1}\left(R_{0}\right)\right)=1$, we have that

$$
\left\langle x^{\prime}, X(\omega)\right\rangle=\Phi\left(x^{\prime}, \omega\right) \text { a.s. } \forall x^{\prime} \in T^{\prime} .
$$

If $x^{\prime} \in F^{\prime}$ and $t_{n}^{\prime} \in T^{\prime}$ such that $t_{n}^{\prime} \rightarrow x^{\prime}$ in $\sigma\left(E^{\prime}, E\right)$, then

$$
\left\langle t_{n}^{\prime}, X(\omega)\right\rangle_{n \rightarrow \infty}\left\langle x^{\prime}, X(\omega)\right\rangle \text { for all } \omega
$$

and an easy argument shows that

$$
\Phi\left(t_{n}^{\prime}, \cdot\right) \underset{n \rightarrow \infty}{\longrightarrow} \Phi\left({x^{\prime}}^{\prime}, \cdot\right) \text { in probability }
$$

and so we have

$$
\Phi\left(x^{\prime}, \omega\right)=\left\langle x^{\prime}, X(\omega)\right\rangle \text { a.s. } \forall x^{\prime} \in F^{\prime}
$$

which proves Lemma 6.1.

Theorem 6.2. Let $X_{1}, X_{2}, \ldots$ be independent, symmetric, B-valued random variables and let $\tau$ be a locally conves Hausdorff topology on $\mathbb{E}$, which is weaker than the norm-topology, and such that the unit ball of $E$ is $\tau$-closed.

Let $S_{n}=\sum_{1}^{\infty} X_{j}$. If $E$ is separable then the following statements are equivalent:

$$
\left(S_{n}\right) \text { converges a.s.; }
$$

$\left(S_{n}\right)$ converges $\tau$-wealily;

$$
\text { . }\left(S_{n}\right) \text { is } \tau \text {-tight; }
$$

$$
\left(S_{n}\right) \text { has a subsequence which converges } \tau \text {-wealcly. }
$$

Proof. It is evident that (6.4) implies (6.5) and (6.6), and that (6.5) implies (6.7), and (6.6) implies (6.7). Hence the only non-trivial implication is that (6.7) implies (6.4).

So let $\left(n_{j}\right)$ be a sequence of integers such that $\left(S_{n_{j}}\right)$ converges $\tau$-weakly. Let $F^{\prime}=(E, \tau)^{\prime}$; then $F^{\prime} \subseteq E^{\prime}$, and since

$$
\left\langle x^{\prime}, S_{n}\right\rangle=\sum_{j=1}^{n}\left\langle x^{\prime}, X_{j}\right\rangle,
$$

we find, from Theorem 17.B, p. 251, in [12], that

$$
\lim _{n \rightarrow \infty}\left\langle x^{\prime}, S_{n}(\omega)\right\rangle=\Phi\left(x^{\prime}, \omega\right)
$$

exists for a.a. $\omega \in \Omega$ and all $x^{\prime} \in F^{\prime}$. And if $\mu$ is the $\tau$-weak limit of $\left(S_{n_{j}}\right)$ then

$$
P\left(\Phi\left(x^{\prime}\right) \epsilon A\right)=\mu\left(x \mid\left\langle x^{\prime}, x\right\rangle \epsilon A\right) \quad \forall x \epsilon^{\prime} F^{\prime} .
$$

Hence, by Lemma 6.1, there exists a random variable $S(\omega)$ such that for all $x^{\prime} \in F^{\prime}$ we have

$$
\left\langle x^{\prime}, S_{n}(\omega)\right\rangle_{n \rightarrow \infty}\left\langle x^{\prime}, S(\omega)\right\rangle \text { a.s. }
$$

Now let $T^{\prime}$ be a countable subset of $A^{\prime}=F^{\prime} \cap\left\{x^{\prime} \in E^{\prime} \mid\left\|x^{\prime}\right\| \leqslant 1\right\}$ which is sequentially $\sigma\left(E^{\prime}, E\right)$-dense in $A^{\prime}$. Then there exists $\Omega_{0} \epsilon \mathscr{F}$, with $P\left(\Omega_{0}\right)=1$, and such that

$$
S_{n}(\omega) \underset{n \rightarrow \infty}{\longrightarrow} S(\omega) \text { in } \sigma\left(E, T^{\prime}\right) \quad \forall \omega \in \Omega_{0} .
$$

Now it is easily seen that $\sigma\left(E, T^{\prime}\right)=\tau_{0}$ is a locally convex Hausdorff topology on $E$ (see (5.8)), such that the unit ball of $E$ is $\tau_{0}$-closed, and $\tau_{0}$ is weaker than the norm-topology. Hence, by Corollary 5.5, we have that $\left(S_{n}\right)$ converges a.s. and so the theorem is proved.

Remarlk. Compare this with Theorem 4.1 in [8]. Ito and Nisio also shows in [8] that $\|\cdot\|$-weak convergence of $\sum_{i}^{\infty} X_{n}$ implies a.s. convergence whenever $\left(X_{n}\right)$ is a sequence of independent $E$-valued random variables.

Added in proof. S. Kwapien has recently proved that my conjecture (5.5) is true (oral communication).

\section{References}

[1] C. Bossaga and A. Pelczyńslii, On bases and unconditional convergence of series, in Banach spaces, Studia Math. 17 (1958), pp. 151-164.

[2] S. D. Chatterjii, Martingale convergence and the Radon-Nikodym theorem in Banach spaces, Math. Seand 22 (1968), pp. 21-41.

[3] E. Dubinsky, A. Pelczyński, and H. P. Rosenthal, On Banach spaces $X$ for which $\Pi_{2}\left(\mathscr{L}_{\infty}, X\right)=B\left(\mathscr{L}_{\infty}, X\right)$, to appear.

[4] N. Dunford and J. T. Schwartz, Linear Operators. Part 1, New York-London 1958.

[5] W. Foller, An introduction to probability theory and its applications, Vol. 2, New York-London-Sydney, 1966.

[9] J. Hoffmann-J ørgensen, The theory of analytio spaces, Matematisk Institut, Aarhus Universitet, Various Publications Series No. 10, 1970.

[7.] A. and C. Tonescu Tulcea, Topies in the theory of liftings, Berlin-HeidelbergNew York, 1969.

[8] K. I to and M. Nisio, On the convergence of sums of independent random variables, Osaka J. Math. 5, (1968), pp. 35-48.

[9] J. P. K ala an o, Series of random functions, Heath Math. Monographs, Lexington, Mass., 1968.

7 - Studia Mathematica LIr.2 
[10] G. Köthe, Topological vector spaces, Berlin-Heidelberg-New York, 1969.

[11] S. Kwapień, Isomorphic characterizations of inner product spaces by orthogonal series with vector-valued coefficients, to appear.

[12] M. Lò̀ve, Probability theory, Princeton, New Jersey-Toronto-New YorkLondon, 1963 (3rd edition).

[13] G. Nordlander, On sign-independent and almost sign-independent convergence in normed linear spaces, Arkiv för Mat. 4, 21 (1961), pp. 287-296.

[14] Y. V. Prohorov, Oonvergence of random processes and limit theorems in probability theory, Teor. Veroyatnost. i. Primenen 1 (1956), pp. 177-238.

[15] B. Rosen, On the asymptotic distribution of sums of independent identically distributed random variables, Arkiv för Mat. 24 (1961), pp. 323-332.

Received October 2, 1973

\section{On Banach spaces containing $c_{0}$}

A supplement to the paper by J. Hoffmann-Jørgensen "Sums of independent Banach space valued random variables"

by

\section{S. KWAPIEN (WarszaWa)}

Abstract. It is proved that a Banach space $E$ does not contain subspaces isomorphic to $c_{0}$ if and only if the almost surely boundedness of sums of independent, symmetric $E$-valued random variables implies the almost surely convergence of the sums.

We shall prove the following result conjectured by Hoffmann-Jørgensen in the preceding paper [2].

THEOREM. For every Banach space $E$ the following conditions are equivalent:

(i) $E$ does not contain subspaces isomorphic to the space $c_{0}$ of all scalarvalued sequences convergent to zero,

(ii) $L_{1}(E)$ does not contain subspaces isomorphic to $c_{0}$,

(iii) the almost surely boundedness of sums of independent, symmetric, $E$-valued random variables implies the almost surely convergence of the sums.

It has been proved in [2] that to prove the theorem it is enough to establish the following

Proposition. Let $E$ be a Banach space. Let $\left(\varepsilon_{i}\right)$ be a Bernouilli sequence on a probability space $(\Omega, \mathscr{B}, P)$, i.e., a sequence of independent random variables such that $P\left(\varepsilon_{i}=1\right)=P\left(\varepsilon_{i}=-1\right)=\frac{1}{2}$ for all $i$. Let $\left(x_{i}\right)$ be a sequence in $E$, such that $\inf _{i}\left\|x_{i}\right\|>0$ and $P\left(\sup _{n}\left\|\sum_{i=1}^{n} \varepsilon_{i} x_{i}\right\|=\infty\right)=0$. Then $E$ contains a subspace isomorphio to $c_{0}$.

Proof. Let $\mathscr{B}_{0}$ denote the $\sigma$-subfield of $\mathscr{B}$ generated by all $\varepsilon_{i}$ s. Then it is easy to see that for $B \in \mathscr{B}_{0}$

$$
\lim _{i} P\left(B \cap\left(\varepsilon_{i}=1\right)\right)=\lim _{i} P\left(B \cap\left(\varepsilon_{i}=-1\right)\right)=2^{-1} P(B) .
$$

Pick $M<\infty$ so that $P(A)>2^{-1}$ where

$$
A=\left\{\omega \epsilon \Omega: \sup _{n}\left\|\sum_{i=1}^{n} \varepsilon_{i}(\omega) x_{i}\right\|<M\right\}
$$

